\title{
A pluriverse of local worlds: a review of Computing within Limits related terminology and practices
}

\author{
Marloes de Valk \\ devalkm@lsbu.ac.uk \\ London South Bank University \\ London, United Kingdom
}

\begin{abstract}
Green capitalism is shaping public discourse on how to best deal with the climate crisis, yet doesn't challenge the 'business as usual' of free market capitalism that caused the crisis in the first place. Small scale practices challenging 'business as usual' aren't part of public discourse because they are small scale, less visible, often hard to access, easily appropriated by and seemingly unable to compete with the powerful lobby of large corporations. With Big Tech having an increasingly negative impact on the environment, and simultaneously shaping the discourse on how to best tackle the climate crisis, it is important to give voice and visibility to these alternatives.

There is a rich diversity of practices and views on how network infrastructures' impact could be lowered. This study aims to make them visible through a mapping of the different terms currently in circulation used by communities of practice, with the aim of teasing out the diverse thinking informing the infrastructures that are developed, maintained and repaired. The mapping will be based on a review of relevant literature and the results from a survey conducted on Mastodon, an open source decentralized social network with a user base that includes many developers and activists working on sustainability and social justice in relation to computing. The mapping aims to celebrate differences and also show what common ground this pluriverse of small scale community practices share.
\end{abstract}

\section{KEYWORDS}

sustainability, communities of practice, degrowth, resilience, repair, minimalism

Reference Format:

Marloes de Valk. 2021. A pluriverse of local worlds: a review of Computing within Limits related terminology and practices. In LIMITS '21: Workshop on Computing within Limits, fune 14-15, 2021.

\section{INTRODUCTION}

This study maps the terminology used by communities of practice to describe alternative network and computing infrastructures informed by ecological ethics and describes the characteristics of associated practices. Big Tech has a growing negative impact on the environment, and is shaping the discourse on how to best deal with

Permission to make digital or hard copies of part or all of this work for personal or classroom use is granted without fee provided that copies are not made or distributed for profit or commercial advantage and that copies bear this notice and the full citation on the first page. Copyrights for third-party components of this work must be honored. For all other uses, contact the owner/author(s).

LIMITS '21, fune 14-15, 2021,

(C) 2021 Copyright held by the owner/author(s). this, without proposing any fundamental change, without challenging the 'business as usual' of free market capitalism, which is at the heart of the problem. This review has the goal of making the many counternarratives to the one of green capitalism visible, show the differences and similarities between them, create bridges between different fields and contexts and demonstrate the wealth this diversity of practices, and the thinking that informs them, brings. In Designs for the Pluriverse [14], Arturo Escobar describes design as an ethical praxis of world making from the perspective of radical interdependence and a pluriversal imagination. His work has informed this paper's emphasis on diversity and plurality, and its resistance to universal and global solutions. In Escobar's words: "If worlds are multiple, then the possible must also be multiple" [14].

The scope of this study is the terminology used by small scale communities running their own network infrastructures and terms directly related to those. The focus on terminology allows us to dive into the different ways of thinking related to each term. It is not a goal to promote the adoption of a specific term, or coin a new one, as this does not align with the idea of pluriversality. We will not cover the terminology associated with the larger fields of Sustainable Human-Computer Interaction (SHCI), ICT for Sustainability (ICT4S), Environmentally Sound Technologies, Crisis Informatics or ICT for Development (ICTD). The links between ICTD and Computing within Limits is discussed in detail by Jay Chen [7].

The terms covered in this paper are Computing within Limits, benign computing, collapse informatics, permacomputing, small technology, salvage computing and low-tech, as well as three historical terms: liberatory technology, appropriate technology and convivial computing. This selection of terms is the result of a literature review and the outcome of a survey on Mastodon, an open source decentralized social network. The ten terms are a selection, future research will expand on this vocabulary with special attention to non-Western practices.

The paper starts with a description of three historical terms dealing with technology based on ecological ethics, and why they have fallen into disuse. Next, we'll discuss two terms stemming from the Computing within Limits community, which has generated extensive, relevant thinking, with related terminology spreading outside of academia, as evidenced by two terms coming up in the Mastodon survey. Lastly we'll describe four key terms that came up in the survey, which are practiced 'in the wild'. In the conclusion we'll highlight what can be learned from this longitudinal view. 
Table 1: Results of a Mastodon survey asking: "What's the best umbrella term for tech that aims at a lower environmental impact (hardware, software and network traffic) and respects privacy?"

\begin{tabular}{cc}
\hline Term & Percentage of 175 votes \\
\hline permacomputing & $31 \%$ \\
appropriate technology & $15 \%$ \\
low tech & $17 \%$ \\
small tech & $37 \%$ \\
\hline
\end{tabular}

\section{RESEARCH METHODS}

\subsection{Poll on Mastodon}

This review is based on the results of a survey conducted on Mastodon, an open source decentralized social network with a user base that includes many developers and activists working on sustainability and social justice in relation to computing. With over 4.4 million users, Mastodon is currently the largest federated social network, consisting of non-commercial, connected yet autonomously administered and self-hosted servers. Each node is community owned and tends to have its own 'culture', often with a specific focus, such as solar punk or computational culture. These specialized communities tend to cluster, with users of different related nodes following each other, making it easy to reach an audience with special knowledge about a specific topic. This, plus the poll feature that was introduced in 2019, make Mastodon a suitable place for this survey. Even though many people interested in sustainability and computing are using Mastodon, sadly the software itself is not particularly sustainable.

The survey consisted of a poll querying: "What's the best umbrella term for tech that aims at a lower environmental impact (hardware, software and network traffic) and respects privacy?" It was a follow-up of an earlier Mastodon post by 'Calcifer' on December 20,2020, asking about an umbrella term which resulted in 14 suggestions: heirloom computing, retrocomputing, alt-computing, small tech, sustainable computing, resilient technology, respectful computing, minimal computing, As Little Tech As Possible, solar punk, permatech, walkaway computing, convivial technology and human centred technology [5].

A total of 175 votes came in during a 3 day period, but more importantly, the poll triggered a discussion that resulted in the suggestion of 18 additional terms: Computing within Limits, resource constrained computing, regenerative computing, inclusive tech, little tech, degrowth, tethics, Minimal Impact Computing (MIC), efficiency driven computing, rustic computing, slow tech, User Centric Computing, collapse informatics, convivial computing, tiny tech, non-coercive tech, consentful tech and smol tech [10]. For this paper, we selected a few key terms to unpack in detail, to give insight into the related thought and philosophies of each, how they differ and most importantly how they relate and help shape a counternarrative that disrupts the master narrative of progress through eternal growth still embodied in green capitalism.

\subsection{Literature review}

In addition to the Mastodon poll, we've conducted a literature review of published research addressing technology design aiming at a smaller environmental footprint. We reviewed historical material in relation to appropriate technology, convivial tools and alternative technology, as well as contemporary material, especially research published in the context of the Computing within Limits workshop.

This study is rooted in a review of research looking at the environmental impact of networked media in the field of media studies. Several scholars from this field have researched the impact of ICT on the environment, from the perspective of 'new materialism' $[6,8,12,37]$. Jennifer Gabrys' Digital Rubbish [19] is notable because of its method of rematerializing electronics by focusing on the ways in which they generate waste, making the multiple ways technology impacts the environment tangible. Jussi Parikka does something similar in A Geology of Media [27], in which he proposes a study of media which entails different spatial and temporal materialisms, tracking chemicals, metals and minerals. Parikka extends media materialism to an ecological agenda and credits artistic practices for doing the hard work of exploring new concepts and narratives before science can.

Sy Taffel's writing places a similar emphasis on experimental praxis as a way forward, encouraging experimentation and creative interventions designed to produce positive biopolitical impacts. His approach to media ecologies as dynamic and complex meshworks, mark chaos and uncertainty produced by noise within complex systems as a way of resisting the type of systemic stasis that accompanies the central claims of 'capitalist realism' [17]. He emphasizes that an abandoning of the reductive and utopian dream of the single glorious revolution that resolves all social and ecological strife everywhere, forever more, does not mean abandoning a struggle for social and ecological justice through multiple molecular revolutions [38]. Taking practices happening at the edge of capitalist production seriously, and a similar celebration of a diversity of ways forward can be found in the work of Anna Tsing [39]. In The Mushroom at the End of the World she argues for the 'arts of noticing' what else is there, besides the story of progress. She describes pericapitalist economic forms as sites for rethinking the unquestioned authority of capitalism. Taffel and Tsing both inform this paper's commitment to the arts of noticing and taking small practices, that do not fit into the rhetoric of progress and scalability, seriously.

In Designs for the Pluriverse, Arturo Escobar [14] describes design as an ethical praxis of world making from the perspective of radical interdependence and a pluriversal imagination. His work is especially valuable in informing this paper's emphasis on diversity, its resistance to universal and global solutions and its valuing of the reimagining and reconstructing of local worlds. His writing describes several practices that embody this approach. While there is plenty of literature analysing the problem of environmental damage caused by network technology, and what approaches could be useful in addressing it, what is not covered in any of the literature I reviewed thus far is a thorough mapping and analysis of the diversity of small communities running network services aiming at a lower environmental impact. 


\section{HISTORY}

There are three historical terms that have informed many contemporary practices: Liberatory technology, appropriate technology and convivial computing. Rooted in anti-technocratic critique, anarchism and proposals for human-scale technologies, they promote ideas of decentralisation, "enoughness" and degrowth that should never have gone out of fashion.

\subsection{Liberatory technology}

The earliest is liberatory technology as used by Murray Bookchin in his 1965 essay Towards a Liberatory Technology [2]. Bookchin describes the possibility of an environmentally-friendly technology, which would "make man's dependence upon the natural world a visible and living part of his culture". Bookchin envisaged small communities integrated into the natural environment and using small-scale technologies which permit decentralisation and autonomy. His article succinctly expresses the vision of a utopian ecological lifestyle, which later became associated with the term "alternative technology" and the Undercurrents magazine in the UK.

The main characteristic of liberatory technology, in line with Bookchin's anarchist politics, is decentralisation, in order to avoid centralised economic and administrative control. It is powered by renewable energy, non-coercive, adapted to specific local needs, small scale, multipurpose in order to avoid underuse and shared among communities. In Ecology and Revolutionary Thought, Bookchin points to the value of organic differentiation, not mechanical standardization, for balance in society and nature alike. His view of technology reflects this, and resonates with current philosophies on sustainable design, such as that of Arturo Escobar as expressed in Designs for the Pluriverse [14], and resonates with the characteristics of benign computing and permacomputing.

\subsection{Appropriate Technology}

In 1973 a second term appeared, in the book Small is Beautiful by economist Ernst Schumacher [33]. He first used intermediate technology, to describe technology designed with special consideration to the environmental, ethical, cultural, social, political, and economic aspects of the community it is intended for. The term changed to Appropriate Technology (AT) and became a movement encompassing technological choice and application that is about 'enoughness', robustness and sustainable living. Although many associated projects involve the introduction of technology to developing countries, it is practised in a variety of contexts.

The movement had its peak in the late seventies to early eighties. After that it was replaced with the term 'Environmentally Sound Technologies'. In 1983, an OECD report described AT as "low investment cost per work-place, low capital investment per unit of output, organizational simplicity, high adaptability to a particular social or cultural environment, sparing use of natural resources, low cost of final product or high potential for employment." [22] Because of its focus on adaptation to the needs of the community it is intended for, it could also be described as decentralised and small scale.
There is somewhat of a resurgence happening, with a growing number of organisations adopting Open Source Appropriate Technology (OSAT) such as the Open Source Ecology project which is working towards a Global Village Construction Set (GVCS), with blueprints "for the easy fabrication of the 50 types of industrial machines that it takes to build a small civilization with modern comforts" [13]. They apply open design principles to AT. A big potential of OSAT is that designs are free as in gratis and as in libre, they can be peer-reviewed with iterative improvements which can stimulate development, increase quality over time and generate adaptations to specific local needs.

\subsection{Convivial computing}

In 1973, Ivan Illich published Tools for Conviviality[21], a book about the proper use of technology. Illich pleads for a pluralism of limited tools that guarantee an individual's right to work with independent efficiency, the development of which is "as unpredictable, creative and lively as the people who use them" [21]. His ideas are rooted in ecological thought. He points to overgrowth and overproduction threatening the right to a livable environment. He imagined a future society both very modern yet not dominated by industry, which recognizes natural scales and limits. Illich's thinking about tools created by and for a community of users was a great influence on Lee Felsenstein, one of the first developers of the personal computer, designer of the Osborne- 1 and member of the Homebrew Computer Club.

A 1987 paper by A. C. Lemke and G. Fisher describes convivial computing as computing in which the user has control over the tool on multiple levels [16]. Convivial computing should give a user a desired amount of control but shouldn't require that it be exercised. In their vision, convivial tools will break down the distinction between programming and using programs and see the distinction between user and programmer as a major obstacle for the usefulness of computers. Convivial tools encourage users to be actively engaged with, and to generate creative extensions to, the artefacts given to them, releasing designers of tools from the impossible task of anticipating all possible uses of a tool and all people's needs.

Characteristics of convivial computing are 'soft software', that is software that can be changed by the user, simple and modular, in order to avoid having to anticipate what users might want, and ease of use for both casual and expert users. Although important to Illich, environmental concerns aren't mentioned by Lemke and Fischer, yet their modular approach based on the selection and combination of existing software components, and the idea of 'soft software' that turns users into designers, makes convivial computing very suitable for use with old hardware and therefore indirectly encourages repair. Convivial tools are also adaptable to changes in, or collapse of, infrastructures, especially if they were combined with open source licenses. The application of Lemke and Fischer's ideas on convivial computing to sustainability would be very much in line with Illich's ideas on degrowth and a modern society of responsibly limited tools. 


\subsection{Falling into disuse}

Even though all three terms have remained extremely relevant, they have fallen into disuse during the $80 \mathrm{~s}$. The main reason is that even though during this decade the collateral damage of industrial capitalism - acid rain, the hole in the ozone layer, global warmingbecame painfully apparent, the rise of neoliberalism made sure the solving of all problems would be left to the market. With shrinking governments, deregulation and unfeathered consumerism and automation on all levels of society fueling economic growth in the Global North, alternative views on the role of technology in society didn't stand a chance. In their essay Appropriate Measures, Jackie Brown and Philippe Mesly point out that social transformation doesn't happen through technology, not without a clear political agenda to accompany it [4]. They point out that AT didn't engage with the reasons why the Global South was struggling and the Global North was overconsuming, and instead aimed at fighting symptoms rather than the disease. Where AT was missing this political agenda, liberatory technology was too political to survive neoliberalism and convivial computing's modularity didn't fit the software industry's growth based business model. Yet many of the ideas developed in the 70 s are now resurfacing under different names.

\section{COMPUTING WITHIN LIMITS}

The limits Illich described have found their way into the title of the Computing within Limits workshop, that has taken place annually since 2015. It has brought together a group of scholars dedicated to promote the design of computing contributing to a transition to a future in which the well-being of humans and other species is the primary objective [24]. A wealth of interesting terms and related design principles have emerged. First the term Computing within Limits itself, which brings together three principles, according to a 2018 paper by Nardi et al.[24]: it questions growth and aims instead for a steady-state economy, it considers models of scarcity in order to promote resilience in a diversity of current and future contexts, and lastly it aims at reducing energy and material consumption while avoiding the Jevons paradox or rebound effect, in which gains in efficiency often result in lower costs, a subsequent growing demand and increased resource consumption. The workshop's focus and the interpretation of its title have shifted a little over the years, starting with an emphasis on designing in the abundant present for the use in a future of limits, to designing for the present with an awareness of current real-world limits. There are two additional terms from past LIMITS workshops that are especially interesting to this mapping: benign computing and collapse informatics.

\subsection{Benign computing}

Benign computing is a term coined by Barath Raghavan in his 2015 paper Abstraction, Indirection, and Sevareid's Law: Towards Benign Computing[29]. It is a general design framework, inspired by appropriate technology, that aims at computing systems that are less likely to produce harm to the ecosystem and subsequently human society. One of its strategies is to avoid becoming trapped by Sevareid's Law, which states that the chief source of problems are solutions. Benign computing critiques and tries to avoid tech solutionism. It aims at foreseeing potential drawbacks, to keep evaluating if the benefits still outweigh those drawbacks, and making sure both benefits and drawbacks are distributed and defined from a diverse range of perspectives. Ultimately benign computing limits structural power, such as that wielded by GAFAM, through systems with a large underlying diversity, like in nature.

The characteristics show a lot of overlap with the three historical examples from the start of this paper but are much more clearly defined and embedded in contemporary design practices. Benign computing is decentralised and can scale-out through federation of autonomous nodes under diverse administrative control. It is resilient and fails well by mimicking nature in the way it handles failure through complexity and diversity. It is based on open design principles, using open source software and hardware licenses to allow for a diversity of implementations within a scale-out system. It is fractal in nature, meaning systems should be decentralised, resilient and use open design on all levels of their structure to ensure these principles can be applied where needed.

\subsection{Collapse informatics}

The last term from the LIMITS archive which is important to mention is collapse informatics, software engineering taking advantage of today's abundance in computing power to prepare for a future in which current infrastructures have collapsed [28,31]. The aim is to develop a set of methods, metrics and tools to design for collapse. Collapse informatics is inspired by ICT4D and SE4S and is the most radical in its characteristics because of its post-collapse target. It is based on decentralisation, not only because current centralised services and networks will break down, but also because peer-to-peer infrastructures are more resilient and flexible. It aims for simplicity-software should function on existing hardware-and modularity in order to enable a diversity of combinations and implementations. Systems need to be resilient to intermittent energy supply and network connectivity. Next to that collapse informatics should prioritize community needs and make use of open source licenses to contribute to a knowledge commons in order to be able to succeed in case of economic collapse.

There are some software and hardware projects that could be considered collapse informatics, even though they do not use the term themselves and might not apply all characteristics described by Penzenstadler et al [28]. Collapse OS is a Forth operating system and collection of tools to preserve the ability to program microcontrollers through civilizational collapse [26]. Collapse OS, as the name suggests, is only an OS and requires very creative post-collapse coding in order to become useful. On the other side of the spectrum is disaster.radio, that promises a disaster-resilient off-grid, solar-powered, long-range mesh network built on free, open source software and affordable, open hardware [11]. It offers a fully functional communication system, but relies on a substantial amount of custom hardware and even 3D printed casing and contains patented and proprietary components, which might not only prove counterproductive in case of an actual disaster, but also begs the question for who this project is affordable. Whatever the shape of the system, what is most interesting is to consider the current state of our planet as one of collapse already, so we can make use of the more radical methods and tools described by Penzenstadler et al. today. 


\section{PERMACOMPUTING}

Permacomputing is a term originating from the demoscene, known for squeezing the most out of very restricted computing resources, such as the $4 \mathrm{k}$ intro with a maximum executable file size of 4096 bytes. Artist programmer Ville-Matias Heikkilä, aka Viznut, coined the term in a text he published on his website in 2020 [20]. What stands out in this writing is the holistic approach to computing and sustainability by taking inspiration from permaculture. In both computing and agriculture, problems are usually solved by increasing control over a process, which often goes hand in hand with an increase in resource use. Permaculture uses methods that lets nature do the work, minimizing the reliance on artificial energy. Heikkilä sees similarities between how both permaculture practitioners and hackers find clever solutions to problems. He writes that the existence of computers can only be justified by their ability to augment the potential of humans to have a strengthening effect on ecosystems.

The text is incredibly rich and detailed, so we'll only highlight a few characteristics. Instead of one dominant technology and linear progress, permacomputing aims at a diversity of approaches developing at all levels. It is enmeshed in culture, because people have a deep connection to technology, beyond the tool, as part of art, ecology, philosophy and history. Permacomputing is accountable, it only does heavy computation if this saves resources elsewhere and uses automation to save humans from repetitive and time consuming tasks when it requires little energy from computers. It values maintenance and encourages programmers to refactor and rewrite programs to keep them small and efficient, instead of counting on Moore's law to compensate for software bloat, something also covered by Barath Raghavan and Daniel Pargman in Refactoring Society: Systems Complexity in an Age of Limits[32]. Instead of planned obsolescence, permacomputing practices planned longevity, reuse and repair of existing technology and approaches waste as a resource. Just like all other terms, it points to decentralisation and modularity so that it can be adapted to suit local community needs. Permacomputing contributes to a commons by placing technology in the public domain and promotes the sharing of resources.

The term got picked up by other artists, programmers and activists, such as by the programmer of Ariane, a Gemini protocol browser for Android, and by the maker of the solar powered Leaf server, but has yet to become more widely used. Similar to liberatory technology and Illich's Tools for Conviviality, the term encompasses political ideas on the role of technology in society, but next to that describes how these ideas might be applied in contemporary design and practice.

\section{SMALL TECHNOLOGY}

Whereas permacomputing sprouted from the mind of one programmer artist and got picked up by others later, small technology, smallnet and smolnet are associated with communities using alternative network infrastructures, delinking from the commercial Internet. They are using alternative networking protocols such as Gopher and Gemini or communicate on a server itself when logged in, instead of through publishing, like on a Public Access Unix System (PAUS or pubnix). Gopher and pubnixes have been around since the late 80 s and early 90 s, and are currently experiencing a revival due to an increasing amount of people feeling frustrated with the state of the commercial Internet, both for privacy and environmental reasons. Even though Gopher fans, pubnix and tildeserver visitors are not necessarily the same crowd, they have some common goals and practices that align with many of the terms mentioned in this paper.

The smallnet crowd is DIY-minded, they self-host communityrun servers and community-built services, often using limited CPU, memory, disk space and bandwidth by choice, using simple protocols, formats and tools. The smallnet uses cohesive and modular tools in classic Unix style and is home to small communities that engage in close communication instead of broadcasting to an anonymous mass [36]. A Gopher enthusiast who goes by the name of Solderpunk has created a protocol called Gemini, that expands Gopher with TLS encryption but is leaner than the web [34]. His protocol makes additional network transactions impossible in order to avoid bloat. The protocol is free of in-line images, external style sheets, fonts, scripts, iframes and more. It is attracting a growing number of people looking for more privacy, a smaller environmental footprint and a more close-knit community. Resource minimalism and the decentralised nature of the smallnet, and the awareness within the community of the links between privacy and environmental impact of technology makes it worth paying attention to within the context of Computing within Limits. This community approaches everyday (network) practice as a site of political struggle. Something Silvia Federici puts forward in Re-enchanting the World: Technology, the Body, and the Construction of the Commons "we are beginning to recognize that the new paradigms may come from those who in fields, kitchens, and fishing villages across the planet struggle to disentangle their reproduction from the hold of corporate power and preserve our common wealth" [15].

\section{SALVAGE COMPUTING}

Something a lot of the terms have in common is an emphasis on resource minimalism and repair, yet many communities trying to lower their environmental footprint are currently seeking this minimalism in hardware that is very hard to repair and is newly produced. Salvage computing is a response to this hype of small low-power single-board computers, aware of the resulting production of yet more electronics while the world is swimming in e-waste. Devine Lu Linvega, one of the voices of the solarpunk merveilles.town Mastodon instance, proposes that creating software targeting old hardware might be a better approach [23]. Gemini creator Solderpunk thinks along similar lines when writing "the real long-term future of computing consists of figuring out how to make the best possible use we can out of the literal millions of devices which already exist" [35]. Scholar Jennifer Gabrys describes salvage as a practice of engaging with the discarded "with an eye to transforming what is exhausted and wasted into renewed resources" [18]. She adds the important observation that this process also means engaging with the conditions that led to disrepair.

The Right to Repair movement, the Restart project, repair cafes, iFixit and U-Fix-It are all targeted at making devices last longer but with the exception of the Right to Repair movement, do not focus on the conditions that led to disrepair: planned obsolescence, the rapid upgrade-or-die cycle of the tech industry and consumer capitalism 
in general, not to mention the impact of this on the Global South, which is receiving the West's e-waste and suffers the pollution caused by the production of the Global North's technology. Out of precarity, and because of the ongoing impact of colonialism, there are very rich and creative repair practices in existence-Jugaad, Gambiarra, Resolver, Shanzhai. Because of the sudden attention in the West to e-waste and supply chains, these practices of improvisation are appropriated and fetishized, yet as Ginger Nolan argues, the romanticizing of the inventiveness of these practices can function as an excuse to keep economic instability and precarity in place [25].

Without romanticizing these practices or ignoring the conditions leading to disrepair, making do with existing and already produced technology saves a lot of resources simply because nothing new needs producing and no e-waste needs processing. As Barath Raghavan and Shaddi Hasan point out in their paper Macroscopically Sustainable Networking: On Internet Quines, a salvage Internet is one way to drastically decrease the Internet's dependencies, removing the need for manufacturing and transportation as it uses only common, locally available components. They acknowledge it cannot be sustained in the long-term [30]. Still, considering today's urgent need to shrink consumption of resources, it is surprising to see that from the list-reduce, reuse, repair and recycle-the last one is the most wasteful, yet has gotten most attention. This emphasis on recycling can only be explained because the other three point to economic degrowth, an unpopular topic in mainstream politics to date. This, again, shows the importance of a political agenda, next to design and praxis. Thanks to the successes of the Right to Repair movement in Europe, repair practices are gaining momentum there. Next to lobbying policy makers, the two most important characteristics of salvage computing are skill sharing and the use and development of open source software that runs on older devices, allowing people to keep using hardware even though the manufacturer has stopped maintaining their product.

\section{LOW-TECH}

Although low-tech originally meant simple and pre-industrial technology, nowadays the term is being used to describe a wide variety of practices that involve some form of technology that has a limited environmental impact. The general aims are accessibility, consideration of the environmental and social impact of a technology and a rejection of technological solutionism, planned obsolescence and consumer capitalism. For example, Phillipe Bihouix, author of L'age des low-tech, describes how France could become a lowtech country instead of a start-up country, questioning the faith in 'green tech', which is supposed to provide a technological fix to the many environmental problems the country is facing. Instead of a return to a pre-industrial age, he proposes an exploration of possible paths towards an economic and industrial system that respects the limits of the planet [1]. Another example is the Low-tech lab project, that was set up in 2015: "At the Low-tech Lab, we use the term 'low-tech' to define the techniques, technologies, services and know-hows that stick to three main principles: Useful. Accessible. Sustainable". Their mission is to show people how to live better with less. Another use of the term, although spelled differently is by Julia Watson, who wrote the book Lo-TEK [41], where TEK stands for Traditional Ecological Knowledge. The book explores naturebased technology, multi-generational knowledge and practices, to celebrate aboriginal innovation instead of discarding it as primitive and isolated from technology.

In relation to network technology, the term appears in the writing of Raghavan and Hasan, who describe a possible low-tech Internet based on simple radios connecting computers without Integrated Circuit boards, again to decrease networking dependencies[30]. Another interesting case is Low-tech Magazine, by Kris de Decker. The online and printed magazine have been around since 2007 and since 2018 a solar powered website, designed and developed by Marie Otsuka and Roel Roscam Abbing, is also available. This solar powered site has been incredibly influential. The aesthetics as well as technical underpinnings have been copied by more and more online publishers, from individual artists to a game festival and a car manufacturer. The different ways the ideas informing the design of the solar website have been implemented by others is worth exploring. First the original idea: "to radically reduce the energy associated with accessing our content" [9]. It is implemented by self-hosting a server completely powered by a solar panel. If there is not enough sunshine, the website will go offline. The creators did not want to maximize uptime, they wanted to reduce energy use. The viewer of the website sees an indicator of how much power is left in the battery powering the server. The design of the website is as simple and open as possible so that it uses as little power as possible both on the server and client side, and is accessible for older devices. To reduce the weight of each page, it is a static site using default typefaces, dithered images and has an off-line reading option.

Because the power usage determined the visual design of the website, it has a very specific aesthetic. More and more sites started copying this style, some from a similar environmental reasoning - aiming at a lightweight site-others stayed on the surface and emulated the design without applying the underlying principles. For its 2021 edition, small indie game festival Now Play This published its website using very similar visual design, using dithered images and a darker background color, but instead of the $561 \mathrm{kB}$ of Lowtech Magazine's homepage, the festival's website downloaded 36.88 MB mostly because of media players and JavaScript running in the background. Another example is Volkswagen Canada that uses ASCII art images and a lightweight design to sell electric cars [40]. It is not so much the web design that is not in line with low-tech philosophy, it's the selling of cars using environmental claims that is pure, yet creative, greenwashing.

A project using Low-tech Magazine's aesthetics that is ideologically more aligned with it, is Solar Protocol by Tega Brain, Alex Nathanson and Benedetta Piantella. It is a web platform hosted across a network of solar-powered Raspberry Pi servers set up in different locations around the world, serving content about the platform itself. When visiting the website, the request is sent to the server which is receiving sunshine at that moment. The visual design of the website also changes depending on which server responds to the request, and how much energy is available. The aim is to add more and more servers to the network, in order to avoid downtime, as the website says: "It's always sunny somewhere!" [3]. The creators call this 'energy-centred design', which focuses on accountability, meaning that for instance computation done to 
generate visualisations on the site are done on the server, rather than on the client-side. That way the content is solar powered and the computational costs are not outsourced to website visitors. The biggest difference between Solar Protocol and Low-tech Magazine is that the latter serves content about the potential of past and often forgotten knowledge and technologies that can be used to design a sustainable society, while the former only publishes about it's own infrastructure. The second difference is that Low-tech Magazine doesn't chase the sun around the globe to maximize uptime, and has an old-fashioned server as a backup when the solar server is down. Both projects use new single-board computers, Solar Protocol needs three while Low-tech Magazine only requires one.

\section{CONCLUSION}

The paper started with three historical terms: liberatory technology, appropriate technology and convivial computing. Even though they remain relevant, they have fallen into disuse because they were either too politically divergent from neoliberal ideology, or not political enough in their approach to local problems with global causes, focusing solely on technology as solution. We've explored some terminology from the Computing within Limits community, an academic context with a reach well beyond academia. Here we've seen a shift from preparing for collapse in the future to thinking of systems respecting planetary boundaries in the present, aiming at reshaping the computing research agenda. Lastly, we've seen four terms from communities practising 'in the wild', that, through their applied philosophies, most clearly demonstrate that similarities in philosophy can still lead to differences in approach.

These differences become most apparent when ideas are implemented in actual software or hardware, in actual praxis. For instance the choice to use old, existing hardware such as in salvage computing or to work with newly produced yet low-power devices like certain low-tech projects. Another example is the choice to develop tools that require a lot of resources but can easily be put together such as disaster.radio; or like Collapse OS, develop tools that require very little hardware but do demand a lot of knowledge and skill from the user with the goal of remaining modular and adaptable to different contexts, whether in the future or simply elsewhere. But these differences are not mutually exclusive and all projects, from the Gemini protocol to solar powered servers, show alternatives to 'business as usual'.

Some projects clearly show the fragility of small scale practices. They are easily appropriated as shown in the case of the Lowtech Magazine aesthetics used by Volkswagen. They can transform into commercial products adding to the amount of hard to repair, short-lived electronics flooding the market, something that lowpower single-board computers are at risk of. Last but not least, these projects are not part of mainstream public discourse, so their ability to trigger new imaginaries of technology that has a smaller environmental footprint remains limited.

The goal of this paper is making the many counternarratives to the one of green capitalism visible, and strengthening them by creating bridges and showing common ground. The most striking similarities are networking practices that are:

- community centred,

- small scale,
- decentralised and autonomously administered,

- modular and multi-purpose,

- resilient through diversity and simplicity (less dependencies),

- resource minimalist (throughout the entire life-cycle of a device), and

- open source or contributing to a commons.

Decentralization deserves to be discussed in more detail because it relates to the value of this study. Decentralised systems allow for a greater adaptability to local needs, economic and administrative autonomy, greater resilience and flexibility than centralised systems. It allows for organic differentiation instead of mechanic standardization, as called for by most of the terms covered. It limits structural power such as that yielded by GAFAM and allows technology to serve a community's needs instead of that of a corporation. Yet decentralisation also brings with it a fragmentation that can render larger societal trends less visible. Academic silos and online communities that are prolific but completely invisible to the larger public make it hard to show the collective effort that is being made. It makes it hard to push back against harmful practices. All terms together are more than the sum of their parts and therefore it is worth creating bridges between them. Not only to show the full width of the different practices in existence, but also to be able to bring them under the attention of policy makers at all levels, because most practices discussed are based on free labor, something that is unsustainable in the long run. This is something to explore in future research.

Next to similarities, there are also differences. The most striking one being the way each term's underlying philosophy engages with their larger political consequences. Some approaches do not question technology being part of the solution and avoid larger political issues altogether (AT), others question the entire organisation of society (liberatory technology), there are those that see everyday practice as the site of political struggle (small technology) and some fall somewhere in between. It is not possible to disentangle a practice or design from its political implications. As Gabrys points out, engaging with repair means engaging with the conditions that led to disrepair [18]. A wider adoption of salvage computing and repair doesn't only rely on a willingness of people to learn how, but also on regulations making sure produced devices are repairable. Small network infrastructure and services based on free labor can only be adopted by a privileged few who have the time and means, and can only be adopted, and adapted, by diverse communities if this labor is valued.

The combined effort of the terms covered brings together the concreteness of practice with the possible frameworks and the imagining of different worlds and politics of philosophy and theory. This longitudinal view hopes to demonstrate the wealth this diversity of practices, and the thinking that informs them, brings. They can enrich each other and together provide a stronger counternarrative: alternatives are possible and already exist. This paper hopes to bring some attention to this pluriverse of local worlds.

\section{ACKNOWLEDGMENTS}

We thank everyone who contributed to the Mastodon poll and thread, and Solderpunk and Viznut for their kind help in search for the origin and first uses of the terms permacomputing and small 
tech. We thank Geoff Cox (London South Bank University, Centre for the Study of the Networked Image), Annet Dekker (University of Amsterdam), Jon Uriarte and Sam Mercer (The Photographers' Gallery). We thank London South Bank University and The Photographers' Gallery for support. We thank Barath Raghavan, Jay Chen and Oliver Bates for their thoughtful feedback.

\section{REFERENCES}

[1] Philippe Bihouix. 2014. L'Âge Des Low Tech: Vers Une Civilisation Techniquement Soutenable. Éditions du Seuil, Paris.

[2] Murray Bookchin. 1986. Towards a Liberatory Technology. In Post-Scarcity Anarchism. Black Rose Books, Montreal, 105-162.

[3] Tega Brain, Alex Nathanson, and Benedetta Piantella. 2021. Solar Protocol. Retrieved May 21, 2021 from http://solarprotocol.net

[4] Jackie Brown and Philippe Mesly. 2021. Appropriate Measures. Retrieved May 21, 2021 from https://reallifemag.com/appropriate-measures

[5] Calcifer. 2020. Sustainable Computing Question on Mastodon. Retrieved May 11, 2021 from https://hackers.town/@calcifer/105409792170000331

[6] Allison Caruth. 2014. The Digital Cloud and the Micropolitics of Energy. Public Culture 26, 2 (73) (2014), 339-364. https://doi.org/10.1215/08992363-2392093

[7] Jay Chen. 2015. Computing within Limits and ICTD. In Proceedings of the First Workshop on Computing within Limits. ACM, Irvine California, 1-6.

[8] Sean Cubitt. 2016. Finite Media: Environmental Implications of Digital Technologies. Duke University Press, Durham.

[9] Kris de Decker. 2018. Low-Tech Magazine. Retrieved May 8, 2021 from https //solar.lowtechmagazine.com

[10] Marloes de Valk. 2021. Sustainable Computing Poll on Mastodon. Retrieved May 1, 2021 from https://post.lurk.org/@103s/105713332161647316

[11] disaster.radio. 2020. Disaster.Radio. Retrieved April 23, 2021 from https://disaster radio/

[12] Paul Dourish. 2017. The Stuff of Bits: An Essay on the Materialities of Information. MIT Press, Cambridge.

[13] Open Source Ecology. 2018. Machines: Global Village Construction Set. Retrieved May 1, 2021 from https://www.opensourceecology.org/gvcs/

[14] Arturo Escobar. 2018. Designs for the Pluriverse. Duke University Press, Durham.

[15] Silvia Federici and Peter Linebaugh. 2019. Re-Enchanting the World: Feminism and the Politics of the Commons. PM Press, Oakland.

[16] Gerhard Fischer and Andreas C. Lemke. 1987. Constrained Design Processes: Steps Towards Convivial Computing. Technical Report. Colorado University at Boulder Department of Computer Science.

[17] Mark Fisher. 2009. Capitalist Realism: Is There No Alternative? Zero Books, Winchester.

[18] Jennifer Gabrys. 2012. Salvage. In Depletion Design: A Glossary of Network Ecologies. Institute of Network Cultures, Amsterdam, 137-140.

[19] Jennifer Gabrys. 2013. Digital Rubbish. University of Michigan Press, Ann Arbor.

[20] Ville-Matias Heikkilä. 2020. Permacomputing. Retrieved May 13, 2021 from http://viznut.fi/texts-en/permacomputing.html

[21] Ivan Illich. 1973. Tools for Conviviality. Harper \& Row, New York.

[22] Nicolas Jequier and Gerard Blanc. 1983. The World of Appropriate Technology. Technical Report. Development Center of the OECD, Paris. 9 pages.

[23] Devine Lu Linvega. 2021. Notes on Longtermism and Sustainability. Retrieved April 10, 2021 from https://wiki.xxiivv.com/site/longtermism.html

[24] Bonnie Nardi, Bill Tomlinson, Donald J. Patterson, Jay Chen, Daniel Pargman, Barath Raghavan, and Birgit Penzenstadler. 2018. Computing Within Limits. In Communications of the ACM (10, Vol. 61). ACM, New York, 86-93. https //doi.org/10.1145/3183582

[25] Ginger Nolan. 2016. Bricolage... or the Impossibility of Pollution. Retrieved May 18, 2021 from https://www.e-flux.com/architecture/structural-instability/ 208705/bricolage-or-the-impossibility-of-pollution/

[26] Collapse OS. 2021. Collapse OS. Retrieved May 10, 2021 from https://collapseos. org

[27] Jussi Parikka. 2015. A Geology of Media. Number 46 in Electronic Mediations. University of Minnesota Press, Minnesota.

[28] Birgit Penzenstadler, Ankita Raturi, Debra J Richardson, M Six Silberman, and Bill Tomlinson. 2015. Collapse (\& Other Futures) Software Engineering. In Proceedings of the First Workshop on Computing within Limits. ACM, Irvine California, 1-3.

[29] Barath Raghavan. 2015. Abstraction, Indirection, and Sevareid's Law: Towards Benign Computing. In Proceedings of the First Workshop on Computing within Limits. ACM, Irvine California, 1-4.

[30] Barath Raghavan and Shaddi Hasan. 2016. Macroscopically Sustainable Networking: On Internet Quines. In Proceedings of the Second Workshop on Computing within Limits (LIMITS '16). Association for Computing Machinery, New York, NY, USA, 1-6. https://doi.org/10.1145/2926676.2926685

[31] Barath Raghavan and Justin Ma. 2011. Networking in the Long Emergency. In Proceedings of the 2nd ACM SIGCOMM Workshop on Green Networking (GreenNets
'11). Association for Computing Machinery, New York, NY, USA, 37-42. https: //doi.org/10.1145/2018536.2018545

[32] Barath Raghavan and Daniel Pargman. 2016. Refactoring Society: Systems Complexity in an Age of Limits. In Proceedings of the Second Workshop on Computing within Limits. ACM, Irvine California, 1-7. https://doi.org/10.1145/2926676. 2926677

[33] Ernst F. Schumacher. 1973. Small Is Beautiful: Economics as If People Mattered. HarperCollins, New York.

[34] Solderpunk. 2019. Project Gemini. Retrieved March 15, 2021 from https: //gemini.circumlunar.space/

[35] Solderpunk. 2020. The Standard Salvaged Computing Platform. Retrieved March 12, 2021 from gopher://zaibatsu.circumlunar.space:70/0/ solderpunk/phlog/thestandard-salvaged-computing-platform.txt

[36] spring. 2019. Small Internet Manifesto. Retrieved March 14, 2021 from gopher://republic.circumlunar.space:70/0/ spring/phlog/2019-01-18_ _Small_Internet_Manifesto.txt

[37] Nicole Starosielski and Janet Walker (Eds.). 2016. Sustainable Media: Critical Approaches to Media and Environment. Routledge, London.

[38] Sy Taffel. 2019. Digital Media Ecologies: Entanglements of Content, Code and Hardware. Bloomsbury Academic, New York.

[39] Anna Lowenhaupt Tsing. 2015. The Mushroom at the End of the World: On the Possibility of Life in Capitalist Ruins. Princeton University Press, Princeton and Oxford.

[40] Volkswagen. 2020. Un Site Ecologique Pour Un Avenir Plus Durable. Retrieved February 24, 2021 from https://www.vw.ca/carbonneutralnet/fr/

[41] Julia Watson. 2021. Lo-Tek: Design by Radical Indigenism. Taschen, Cologne. 